\title{
Cytomegalovirus Infection of the Colon Presenting as a Mass-Like Lesion
}

\author{
Christopher B. Tan ${ }^{a} \quad$ Magdalene Vardaros ${ }^{b}$ \\ Apsara Prasad $^{\mathrm{b}}$ Sadat Rashid $^{\mathrm{b}}$ Keith Dahl $^{\mathrm{b}}$ \\ Duane Moise $^{b}$ Wondwoosen Gebre ${ }^{c}$ Kaleem Rizvon ${ }^{b}$ \\ Paul Mustacchiab \\ Departments of a Medicine, ${ }^{\mathrm{b}}$ Gastroenterology and ${ }^{\mathrm{c}}$ Pathology, Nassau \\ University Medical Center, East Meadow, N.Y., USA
}

\section{Key Words}

Cytomegalovirus infection $\cdot$ Colon $\cdot$ Mass-like lesion

\begin{abstract}
Cytomegalovirus (CMV) is responsible for the most common opportunistic infections in patients with acquired immunodeficiency syndrome (AIDS). The colon is a common site for these infections in patients positive for human immunodeficiency virus. CMV rarely presents as an intraluminal inflammatory mass in the colon. Here we report the case of a CMV infection presenting as a mass-like lesion in an AIDS patient. The clinical diagnosis of CMV infection is largely based on the characteristic endoscopic appearance. Endoscopic procedures are encouraged early in the course of $\mathrm{CMV}$ infection in immunocompromised patients. CMV pseudotumors along with malignancy should be part of the endoscopic evaluation in patients with AIDS.
\end{abstract}

\section{Introduction}

Cytomegalovirus (CMV) is an enveloped, double-stranded, Herpesviridae DNA virus that commonly infects immunocompetent and immunocompromised individuals $[1,2]$. It is transmitted from person to person by saliva, respiratory droplet, breast milk, blood transfusion, sexual contact and organ transplantation [1,3]. Primary CMV infection occurs at any age. CMV establishes latent infection in the myeloid precursor cells of the bone marrow after the initial inciting event. In the normal host, it remains suppressed and under control by the strong virus-specific $\mathrm{CD} 4+\mathrm{T}$ cell and $\mathrm{CD} 8+\mathrm{T}$ cell responses. Reactivation of latent infection occurs in patients with impaired $\mathrm{T}$ cell function as a 
result of transplantation, acquired immunodeficiency syndrome (AIDS), or immunosuppressive treatment [1]. CMV infection induces a transient viremia by which it reaches all parts of the body, subsequently creating a wide spectrum of diseases, including retinitis, colitis, myocarditis, pneumonia, infectious mononucleosis, congenital disease and acute renal failure in renal transplant patients [1,3]. Although this agent rarely causes morbidity in normal immunologic hosts, its virulence is quite different in patients with AIDS [2].

The prevalence of gastrointestinal involvement with CMV in AIDS ranges from 30 to $40 \%[4,5]$. It has the ability to affect the entire gastrointestinal tract from the esophagus to the rectum and may include the pancreas, liver and gallbladder [4]. Clinical presentation of CMV infection in the gastrointestinal tract depends on the extent and the location of the pathologic lesions. Gastrointestinal CMV most commonly manifests as erosive colitis and esophagogastritis with chronic diarrhea, fever, abdominal pain, weight loss, melena and hematochezia [6, 7]. Perforation, hemorrhage and toxic megacolon have also been reported. CMV-induced gastrointestinal pseudotumors in AIDS usually present with abdominal pain in $71 \%$ of cases and with fever, change of bowel habits, weight loss and palpable mass in the remaining. About a third of CMV pseudotumors presented as an obstructing mass in a recent case series [7].

Most gastrointestinal CMV infections manifest as ulcerative lesions, but non-ulcerative lesions have also been reported [8]. Kaposi's sarcoma and non-Hodgkin's lymphoma are the most common intraluminal lesions encountered in AIDS. However, a less common cause of mass-like lesion in AIDS can be induced by CMV [9]. Here we present the unique case of an intraluminal colonic mass caused by CMV infection in an AIDS patient.

\section{Case Report}

A 67-year-old male with a past medical history of prostate carcinoma, osteoporosis, esophageal candidiasis and recently diagnosed AIDS was referred to our emergency department for dizziness and syncope. He had experienced persistent dizziness and an episode of syncope a day prior to admission associated with easy fatigability but denied any abdominal pain, blood per rectum, diarrhea, unintentional weight loss and/or anorexia. Vital signs at admission showed a blood pressure of $92 / 63 \mathrm{~mm} \mathrm{Hg}$, a pulse of $104 / \mathrm{min}$, a respiratory rate of $16 / \mathrm{min}$ and a temperature of $98.1^{\circ} \mathrm{F}$. Physical examination was remarkable for pallor, oral thrush and a 2/6 systolic murmur. Digital rectal examination did not reveal any mass lesions or gross blood. Laboratory results were significant for anemia with a hemoglobin of $5.2 \mathrm{~g} / \mathrm{dl}$ and a hematocrit of $15.2 \%$. Stool occult blood was positive for heme-reducing substances. His most recent plasma values were a human immunodeficiency virus (HIV) RNA level of 42,219 copies/ml and CD4 count of $24 / \mathrm{mm}^{3}$. He was not on highly active antiretroviral therapy (HAART) at that time.

The patient responded to fluid resuscitation and was monitored in the medical intensive care unit. He was scheduled for esophagogastroduodenoscopy (EGD) and colonoscopy to investigate the source of bleeding. Two units of packed red blood cells were transfused prior to the procedure. EGD showed healing esophageal erosions, gastric erosions and erythematous antrum. Colonoscopy

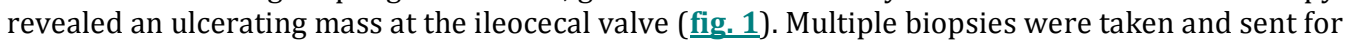
histopathologic examination. Due to a high suspicion of a possible neoplastic process, a computed axial tomography scan of the abdomen and pelvis with contrast was done. It revealed a $3.8 \times 4.5 \times 11 \mathrm{~cm}$ mass involving the cecum and ascending colon, with marked eccentric wall thickening and peripheral enhancement (fig. 2). At least five discrete hypodense liver lesions suspicious for metastases were also noted. Surgical pathologic results of the mass came back as 
fragments of granulation tissue with inflammatory changes highly suggestive of CMV infection (fig. 3 ). The characteristic basophilic inclusion bodies ('owl's eye') were demonstrated within the granulation tissue on histologic examination (fig. 4). Special staining for acid-fast bacilli and fungi was negative.

The patient was started on HAART treatment from which he developed an immune reconstitution inflammatory syndrome against the CMV virus with a subsequent increase of the CD4 count to $284 / \mathrm{mm}^{3}$ and a reduction of the viral load to $<48$ copies $/ \mathrm{ml}$. A repeat CT scan and follow-up colonoscopy weeks later did not reveal any colonic mass ( $\underline{\text { fig. }} \mathbf{5}$ ).

\section{Discussion}

Intraluminal mass lesions in AIDS are usually related to neoplastic and infectious processes. Kaposi's sarcoma accounts for $60 \%$ of malignancies, non-Hodgkin's lymphoma being the next most common etiology (35\%). Infections with mycobacteria or fungi have also been seen to cause mass-like lesions in the gastrointestinal tract on very rare occasions $[4,6]$. CMV-associated mass lesion in AIDS is an uncommon entity. In a review done by Kelesidis et al. in 2010 [7], there were only 15 cases of CMV pseudotumors published in the English literature since 1980 [9]. They have been increasingly observed though over the past decade during the advent of HAART therapy as survival of these patients has improved. CMV-associated mass lesions have been observed throughout the gastrointestinal tract but are most commonly seen in the right colon and the ileocecal valve, similar to our case [4].

The exact pathophysiology of CMV pseudotumors is not clearly understood [10]. The virus has a particular affinity for the gastrointestinal tract, especially the ileum and the right colon [11]. It has been postulated that this affinity is associated with its strong predilection to vascular endothelial cells causing platelet thrombi formation and vascular endothelial thickening with subsequent stromal reaction in the lamina propria, thereby resulting in a mass-like lesion often mistaken as a neoplastic process $[4,10]$. It is important to note that the predominant cells involved in CMV inclusions are stromal and epithelial cells, whereas in CMV-induced ulceration, the predominant cells are endothelial in origin [7].

The diagnosis of gastrointestinal CMV infection is highly dependent on endoscopic findings. In a study by Klatt and Shibata [8], premortem diagnosis of CMV infection in AIDS appears to be greatly dependent on endoscopic sampling of the lungs and the gastrointestinal tract. The endoscopic appearance of CMV infection ranges from focal or diffuse mucosal erythema to extensive, deep, friable ulcerations with non-purulent, clearly defined borders [6]. It is recognized in detail as a well-circumscribed ulceration with persistent inflammation and overlying fibrotic changes $[12,13]$.

The pathologic specimen from tissue biopsy of CMV-infected cells involves a central density of eosinophilic inclusion with a surrounding halo, the characteristic 'owl's eye' intranuclear inclusion bodies [8, 13, 14]. Although herpes and adenovirus infection may also produce similar inclusion bodies, the cells are not as large and do not produce the characteristic cytoplasmic basophilic bodies seen in CMV. If the pathologic diagnosis is doubtful, an immunohistochemical stain using CMV monoclonal antibodies can provide adequate differentiation [8]. Together with biopsy, immuohistochemical staining is considered the gold standard in the diagnosis of gastrointestinal CMV $[7,15]$. 
Although viral serologies such as a high CMV IgM or rising CMV IgG antibodies are useful diagnostic tests for immunocompetent patients, they are not recommended in immunocompromised patients due to lack of reliability and correlation with the organs involved. Moreover, $90 \%$ of AIDS patients are exposed to CMV infection and have had a positive antibody in the past, making serologic testing unhelpful. Detection of viral nucleic acid by polymerase chain reaction (PCR) has also been widely used to demonstrate the presence of CMV. It has fallen out of favor since CMV infection is capable of lifelong latency, and a positive PCR would not always suggest CMV pathogenicity. The same is true for CMV cultures in which the organism can be isolated from human embryonic fibroblast but does not imply active CMV disease [14].

Cellular immunity plays a central role in the control of CMV in healthy and immunocompromised individuals. Several studies have shown that immune reconstitution of specific $\mathrm{T}$ cell responses to a number of pathogens, including $\mathrm{CMV}$, can occur following a HAART regimen [16-19]. In a study by Deayton et al. [16] on HIV patients, CMV DNA levels in the blood rapidly declined after the initiation of HAART. The group further concluded that HAART effectively inhibits CMV replication to as much as $85 \%$, with a median response rate of $61 \%$. This response serves as an effective antiviral activity against CMV infection. Another study by Weinberg et al. [18] on reconstitution of CMV in HIV showed similar results. Our patient had the same immune reconstitution against the CMV virus after initiation of HAART, as evidenced by the disappearance of the mass in CT and colonoscopy despite no specific treatment for CMV.

Pseudotumors related to CMV must be included in the differential diagnosis of AIDS patients presenting with gastrointestinal symptoms along with Kaposi's sarcoma and non-Hodgkin's lymphoma. Endoscopic procedures with multiple biopsies along with immunohistochemical staining remain the cornerstone for diagnosis of gastrointestinal CMV. Furthermore, one should not stop after the identification of a single pathogen, since multiple pathogens can coexist in an immunocompromised host [20]. Physicians must hold a high suspicion for CMV pseudotumors since medical treatments have proven to be highly effective. Moreover, HIV patients with CMV masses have a grim prognosis, and a poorer outcome if left untreated [7].

\section{Disclosure Statement}

The authors declare that they have no competing interests. 


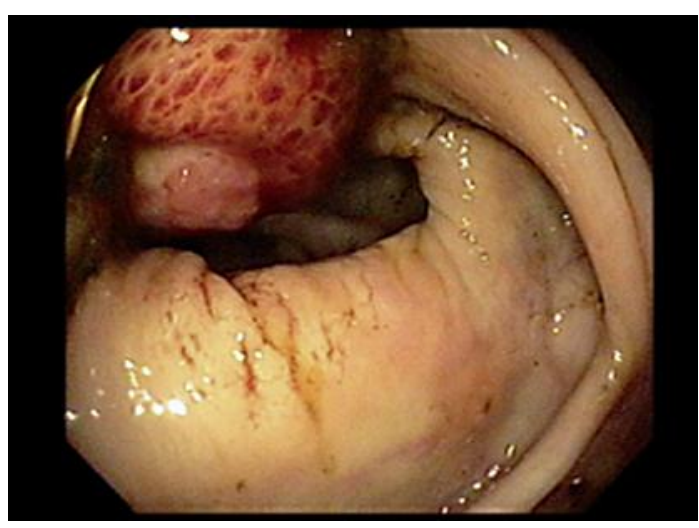

Fig. 1. Colonoscopy showing an ulcerating mass at the ileocecal valve.

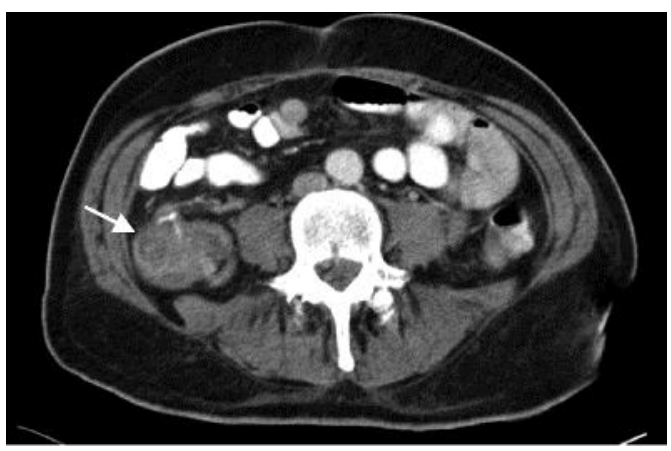

Fig. 2. Computed axial tomography of the abdomen and pelvis showing a large cecal mass measuring $3.8 \times 4.5 \times 11 \mathrm{~cm}$ (arrow).

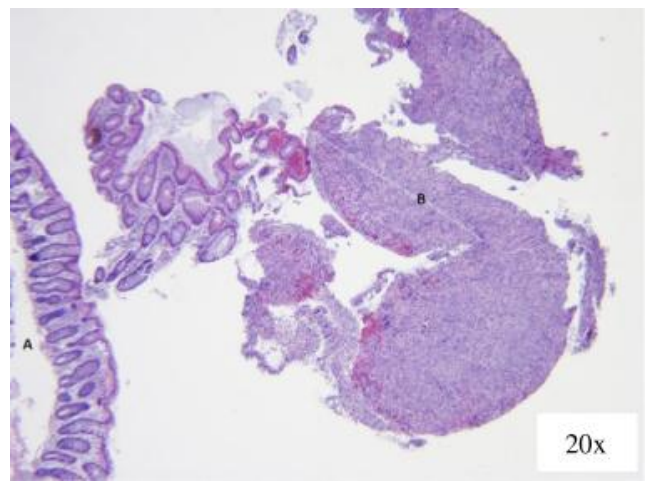

Fig. 3. A Hematoxylin and eosin stain of the mass-like lesion (20× magnification). Note the normal-appearing colonic mucosa with preserved glandular tissues. B The mass-like lesion with active areas of inflammation and complete distortion of glandular tissues. 


\begin{tabular}{r|l|l|l}
$\begin{array}{r}\text { Case Reports in } \\
\text { Gastroenterology }\end{array}$ & $\begin{array}{l}\text { Case Rep Gastroenterol 2012;6:266-272 } \\
\text { DOI: 10.1159/000338842 }\end{array}$ & $\begin{array}{l}\text { Published online: } \\
\text { May 10, 2012 }\end{array}$ & $\begin{array}{l}\text { @ 2012 S. Karger AG, Basel } \\
\text { ISSN 1662-0631 } \\
\text { www.karger.com/crg }\end{array}$ \\
\hline
\end{tabular}

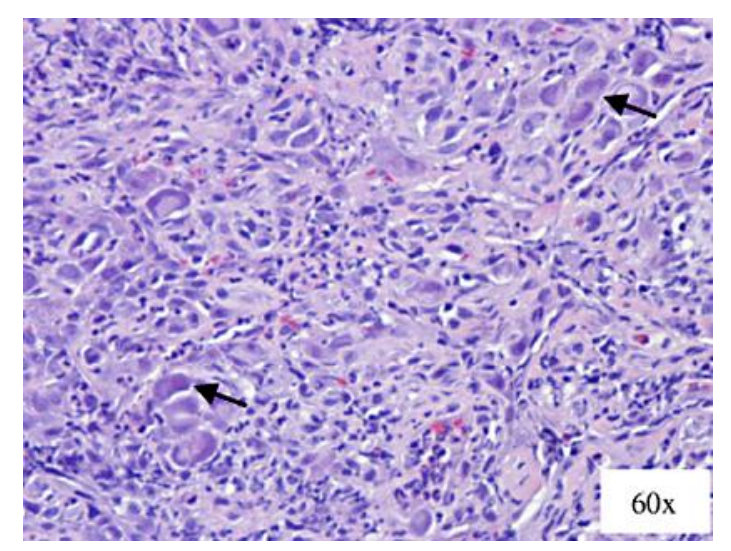

Fig. 4. Hematoxylin and eosin stain of the same mass (60× magnification). Note the basophilic inclusion bodies 'owl's eye' appearance, characteristic of CMV infection (arrows).

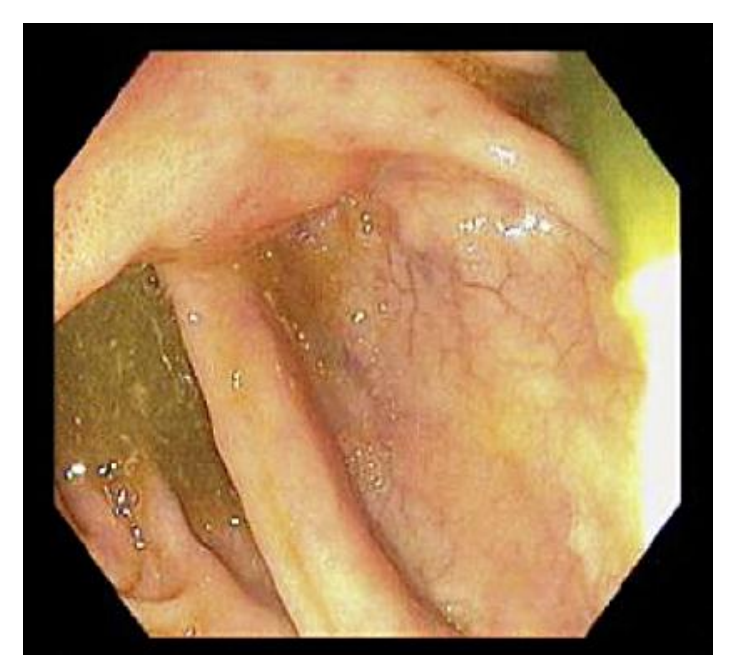

Fig. 5. Follow-up colonoscopy. Note the complete resolution of the mass at the ileocecal junction.

\section{References}

1 Carmichael A: Cytomegalovirus and the eye. Eye (Lond) 2012;26:237-240.

-2 Elta G, et al: A submucosal antral mass caused by cytomegalovirus infection in a patient with acquired immunodeficiency syndrome. Am J Gastroenterol 1986;81:714-717.

3 Drew WL: Cytomegalovirus; in Goldman L, Ausellio D (eds): Cecil Medicine, ed 23. Philadelphia, Saunders Elsevier, 2007, chap 399.

-4 Swansiger B, Orchard JL: A colonic mass lesion due to cytomegalovirus in an immunocompromised patient. J Clin Gastroenterol 1996;22:41-44.

5 Von Both U, et al: Acute cytomegalovirus colitis presenting during primary HIV infection: an unusual case of an immune reconstitution inflammatory syndrome. Clin Infect Dis 2008;46:e38-e40.

6 Rich JD, et al: Discrete gastrointestinal mass lesions caused by cytomegalovirus in patients with AIDS: report of three cases and review. Clin Infect Dis 1992;15:609-614.

7 Kelesidis T, et al: Cytomegalovirus pseudotumor of the duodenum in a patient with AIDS: an unrecognized and potentially treatable clinical entity. Int J Infect Dis 2010;14:e274-e282. 
8 Klatt EC, Shibata D: Cytomegalovirus infection in the acquired immunodeficiency syndrome. Clinical and autopsy findings. Arch Pathol Lab Med 1988;112:540-544.

9 Rajan DP Jr, et al: Cytomegalovirus infection presenting as a colonic mass in a patient with acquired immunodeficiency syndrome. Int Med Case Rep J 2011;20:55-58.

10 Francis ND, et al: Cytomegalovirus infection in gastrointestinal tracts of patients infected with HIV-1 or AIDS. J Clin Pathol 1989;42:1055-1064.

11 Wisser J, et al: Cytomegalovirus pseudotumor presenting as bowel obstruction in a patient with acquired immunodeficiency syndrome. Am J Gastroenterol 1992;87:771-774.

12 Chen C, et al: Colon cytomegalovirus infection: a case report with rare endoscopic presentations. Taiwan Soc Intern Med J 2008;19:67-71.

13 Wilson SE, et al: Acquired immune deficiency syndrome (AIDS). Indications for abdominal surgery, pathology, and outcome. Ann Surg 1989;210:428-433; discussion 433-434.

14 Chetty R, Roskell D: Cytomegalovirus infection in the gastrointestinal tract. J Clin Pathol 1994;47: 968-972.

15 Werneck-Silva AL, Prado IB: Role of upper endoscopy in diagnosing opportunistic infections in human immunodeficiency virus-infected patients. World J Gastroenterol 2009;15:1050-1056.

16 Deayton JR, et al: Rapid reconstitution of humoral immunity against cytomegalovirus but not HIV following highly active antiretroviral therapy. AIDS 2002;16:2129-2135.

17 Komanduri KV, et al: Restoration of cytomegalovirus-specific CD4+ T-lymphocyte responses after ganciclovir and highly active antiretroviral therapy in individuals infected with HIV-1. Nat Med 1998;4:953-956.

18 Weinberg A, et al: Inconsistent reconstitution of cytomegalovirus-specific cell-mediated immunity in human immunodeficiency virus-infected patients receiving highly active antiretroviral therapy. J Infect Dis 2001;184:707-712.

19 Wolf T, et al: A case of severe CMV-colitis in an HIV positive patient despite moderate immunodeficiency. Scand J Infect Dis 2003;35:904-906.

20 Edwards P, et al: The gastrointestinal manifestations of AIDS. Aust N Z J Med 1990;20:141-148. 